\title{
Simple operation sequences to couple and interchange quantum information between spin qubits of different kinds
}

\author{
Sebastian Mehl ${ }^{1,2, \text { * } \text { and David P. DiVincenzo }}{ }^{1,2}$ \\ ${ }^{1}$ JARA-Institute for Quantum Information, RWTH Aachen University, D-52056 Aachen, Germany \\ ${ }^{2}$ Peter Grünberg Institute (PGI-2), Forschungszentrum Jülich, D-52425 Jülich, Germany
}

(Dated: June 1, 2022)

\begin{abstract}
Efficient operation sequences to couple and interchange quantum information between quantum dot spin qubits of different kinds are derived using exchange interactions. In the qubit encoding of a single-spin qubit, a singlet-triplet qubit, and an exchange-only (triple-dot) qubit, some of the singlequbit interactions remain on during the entangling operation; this greatly simplifies the operation sequences that construct entangling operations. In the ideal setup, the gate operations use the intraqubit exchange interactions only once. The limitations of the entangling sequences are discussed, and it is shown how quantum information can be converted between different kinds of quantum dot spin qubits.
\end{abstract}

\section{INTRODUCTION}

Small arrays of singly occupied quantum dot (QD) qubits are now fabricated in GaAs and silicon with great reliability ${ }^{112}$ These setups are of high interest for quantum computation because the electron spin can be used as a qubit .3 Besides the single-spin qubit encoding, also more advanced qubit encodings have been suggested. Most promising are the singlet-triplet qubit (STQ $)^{4}$ and the exchange-only qubit $!^{5}$ These qubits encode quantum information in the $s_{z}=0$ spin subspace of a two-electron double QD (DQD) or in two of the eight possible spin configurations of a three-electron triple QD (TQD).

For all the described qubits, single-qubit gates have been realized with high fidelities. Electric ${ }^{6 / 7}$ or magnetic ${ }^{8} 10$ field pulses can nowadays control single spins with very high fidelities. High-fidelity gates for STQs are also possible when the electron configuration of the DQD is modified while the magnetic field across the DQD is inhomogeneous ${ }^{[11}$ Experimentally, a preparation of the nuclear magnetic field ${ }^{12 \mid 13}$ or a micromagnet ${ }^{14}$ created such static magnetic field configurations. The three-electron TQD can be operated using exchange in-

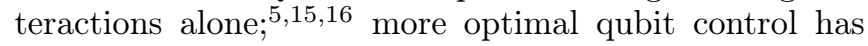
been realized if some of the exchange interactions are not reduced to zero 17118 Two-qubit gates have been proposed for all the qubit encodings using exchange couplings, ${ }^{3 / 5 / 1920}$ while experiments have realized these gates only for single-spin qubits. ${ }^{21}$ STQs or exchangeonly qubits can be coupled indirectly via their charge sector, e.g., using Coulomb interactions ${ }^{22}$ or couplings via cavity modes. ${ }^{17 / 23 \mid 24}$ These approaches have not been successful yet due to a high amount of dephasing that is caused by charge noise. 25

The present study assumes that universal qubit control is possible for the encoded qubits, while two QDs from different qubits are exchange coupled. Operation sequences for entanglement generation and qubit conversion are derived between QD qubits of different kinds. The operation sequences profit from always-on single qubit Hamiltonians during the entanglement sequences, as in earlier studies of TQDs ${ }^{20 \mid 29}$ For STQs, the magnetic fields at the QDs should be prepared independently. Their values need to differ anyway to realize single-qubit control. For the exchange-only qubit, a linear TQD arrangement is considered. Here, the exchange couplings between the neighboring pairs of QDs remain always at similar magnitudes. Such setups have been used in a previous experiment, $\frac{17 \mid 18}{11}$ and can be realized if the occupation of the middle QD is made unfavorable by increasing its chemical potential compared to the outer QDs. $17 / 18$

The main finding of this paper are explicit operation sequences to entangle QD qubits of different kinds. The always-on single-qubit couplings greatly simplify the operation sequences because they reduce the possibility of leakage from the computational subspace. Effective Hamiltonians and entangling sequences are derived; the setups only require two operation sequences to entangle a single-spin qubit and a STQ (or an exchange-only qubit and a STQ), or four operation sequences to entangle a single-spin qubit and an exchange-only qubit. It is shown how the entanglement sequences can be used to swap quantum information between the qubits, and the limitations of the operation sequences are discussed.

The simplicity of the entangling operations shows that a large lattice of QD qubits does not necessarily need to contain identical types of coded qubits (cf., e.g., the description of large scale quantum computation with STQs in Ref. [30]). One can easily convert and couple different QD qubits using the operation sequences derived in this paper. As a consequence, it is possible to use a qubit encoding just for the situation when it is most optimal. It is known that single-spin qubits have exceptionally long coherence times, which makes them an ideal quantum memory ${ }^{[831}$ Encoded spin qubits, like the STQ or the exchange-only qubit, can be employed in their orbital sector, which makes them more ideal for readout or for long-distance couplings. ${ }^{1122}$ It is also possible to use the described operation sequences to couple QD spin qubits to other spin qubits, like, e.g., donor-bound spin qubits. ${ }^{32}$ The electron spin bound to a donor atom is a well-known qubit candidate with many impressive experiments of co- 


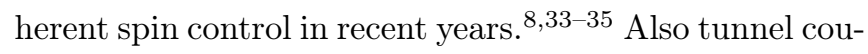
plings between donor-bound and gate-defined spin qubits were shown recently

The organization of the paper is as follows. Sec. II introduces the mathematical descriptions of the single-spin qubit, the STQ, and the exchange-only qubit. Sec. III derives the operation sequences to entangle QD qubits of different qubit encodings. Sec. IV discusses the limitations of these operations and describes how quantum information is converted between different qubits. Finally, the results of the paper are summarized.

\section{QUBIT DEFINITIONS}

\section{A. Single-spin qubit}

A single spin defines a qubit using the states $|0\rangle=|\uparrow\rangle$ and $|1\rangle=|\downarrow\rangle \stackrel{3}{n}$ Universal qubit control is realized when a magnetic field can be tilted to two different directions. The control mechanisms to manipulate spins are magnetic field pulses, ${ }^{9 / 38}$ moving spins in static magnetic fields with spin-orbit interactions, ${ }^{39}$ and driving spins through areas of different magnetic fields! $\frac{6|40| 41}{}$ Without further discussing the exact mechanism, it is assumed here that the magnetic field direction can be rotated to the $\mathrm{z}$ and $\mathrm{x}$ directions to generate rotations around the $\mathrm{z}$ and $\mathrm{x}$ axes of the Bloch sphere. These single-qubit gates are labeled $\mathrm{Z}_{\phi}=e^{-i 2 \pi \frac{\phi}{2} \sigma_{z}}$ and $\mathrm{X}_{\phi}=e^{-i 2 \pi \frac{\phi}{2} \sigma_{x}}$, where $\sigma_{z}=|\uparrow\rangle\langle\uparrow|-| \downarrow\rangle\langle\downarrow|$ and $\sigma_{x}=|\uparrow\rangle\langle\downarrow|+| \downarrow\rangle\langle\uparrow|$ are the Pauli operators. The phase accumulation $\phi=\frac{E_{z}}{h}$ $\left(\phi=\frac{E_{x}}{h}\right)$ is caused by the Zeeman energy $E_{z}=g \mu_{B} B_{z}$ $\left(E_{x}=g \mu_{B} B_{x}\right)$ of the magnetic field in the $\mathrm{z}$ direction (x direction) $\underline{42}^{4}$

\section{B. Singlet-triplet qubit}

STQs are coded using the $s_{z}=0$ spin subspace of a two-electron $\mathrm{DQD} \stackrel{4}{4} \mathrm{QD}_{1}$ and $\mathrm{QD}_{2}$ label the individual QDs of the DQD. Ideally, the electrons are spatially separated, and each QD is occupied with one electron. The logical qubit states are defined by $|0\rangle=|\uparrow \downarrow\rangle$ and $|1\rangle=|\downarrow \uparrow\rangle$, where the first entry labels the electron at $\mathrm{QD}_{1}$, and the second entry labels the electron at $\mathrm{QD}_{2}$. Single-qubit control is realized using a magnetic field gradient between the QDs, corresponding to energy differences $\frac{\Delta E_{z}}{2}\left(\sigma_{z, 1}-\sigma_{z, 2}\right)$, with $\Delta E_{z}=\left(E_{z, 1}-E_{z, 2}\right) / 2$, and the exchange interaction between the QD electrons $\frac{J_{12}}{4}\left(\boldsymbol{\sigma}_{1} \cdot \boldsymbol{\sigma}_{2}-\mathbf{1}\right) . \boldsymbol{\sigma}_{i}=\left(\sigma_{x, i}, \sigma_{y, i}, \sigma_{z, i}\right)$ is the vector of Pauli matrices at $\mathrm{QD}_{i}$.

$\Delta E_{z}$ is usually static in experiments, but $J_{12}$ can be tuned within subnanoseconds by controlling the tunnel coupling or the potential difference of the QDs $\underline{43}$ The magnetic field gradient generates rotations around the $\mathrm{z}$ axis of the Bloch sphere $\mathrm{Z}_{\phi}=e^{-i 2 \pi \frac{\phi}{4}\left(\sigma_{z, 1}-\sigma_{z, 2}\right)}$, with $\phi=2 \Delta E_{z} / h$, and rotations around the $\mathrm{x}$ axis are caused by the exchange interaction $\mathrm{X}_{\phi}=e^{-i 2 \pi \frac{\phi}{4}\left(\boldsymbol{\sigma}_{1} \cdot \boldsymbol{\sigma}_{2}-\mathbf{1}\right)}$, with $\phi=J_{12} / h$. To reduce the leakage probability, experiments are always done at global magnetic fields $\frac{E_{z}}{2}\left(\sigma_{z, 1}+\sigma_{z, 2}\right)$, with $E_{z}=\left(E_{z, 1}+E_{z, 2}\right) / 2$, that lift the degeneracy between the leakage states $\{|\uparrow \uparrow\rangle,|\downarrow \downarrow\rangle\}$ and the computational subspace $\{|0\rangle,|1\rangle\}$.

\section{Exchange-only qubit}

The exchange-only qubit is coded using the $S=\frac{1}{2}$, $s_{z}=\frac{1}{2}$ subspace of three electrons. $\frac{[5}{5}$ The following operations require the initialization to a subspace encoding. ${ }^{44}$ In any case, applying strong, global external magnetic field eases controlled state initializations. The three singly-occupied QDs are labeled by $\mathrm{QD}_{1}, \mathrm{QD}_{2}$, and $\mathrm{QD}_{3}$. The qubit states are defined by $|0\rangle=\frac{1}{\sqrt{2}}(|\uparrow \uparrow \downarrow\rangle-|\downarrow \uparrow \uparrow\rangle)$ and $|1\rangle=\frac{1}{\sqrt{6}}(|\uparrow \uparrow \downarrow\rangle+|\downarrow \uparrow \uparrow\rangle)-\sqrt{\frac{2}{3}}|\uparrow \downarrow \uparrow\rangle$, with the spin labels $\left|\sigma_{\mathrm{QD}_{1}}, \sigma_{\mathrm{QD}_{2}}, \sigma_{\mathrm{QD}_{3}}\right\rangle$. The sum of the exchange interactions $\frac{J}{4}\left[\left(\boldsymbol{\sigma}_{\mathbf{1}} \cdot \boldsymbol{\sigma}_{2}-\mathbf{1}\right)+\left(\boldsymbol{\sigma}_{\mathbf{2}} \cdot \boldsymbol{\sigma}_{\mathbf{3}}-\mathbf{1}\right)\right]$, with $J=\left(J_{12}+J_{23}\right) / 2$, and their difference $\quad \frac{\Delta J}{4}\left[\left(\boldsymbol{\sigma}_{\mathbf{1}} \cdot \boldsymbol{\sigma}_{\mathbf{2}}-\mathbf{1}\right)-\left(\boldsymbol{\sigma}_{\mathbf{2}} \cdot \boldsymbol{\sigma}_{\mathbf{3}}-\mathbf{1}\right)\right], \quad$ with $\Delta J=\left(J_{12}-J_{23}\right) / 2$, provide universal control of the subspace $\{|0\rangle,|1\rangle\}$. $J$ causes a rotation around the $\mathrm{z}$ axis of the Bloch sphere $\mathrm{Z}_{\phi}=e^{-i 2 \pi \frac{\phi}{4}}\left[\left(\boldsymbol{\sigma}_{1} \cdot \boldsymbol{\sigma}_{\mathbf{2}}-\mathbf{1}\right)+\left(\boldsymbol{\sigma}_{\mathbf{2}} \cdot \boldsymbol{\sigma}_{\mathbf{3}}-\mathbf{1}\right)\right]$, with $\phi=J / h$, and $\Delta J$ causes a rotation around the $\mathrm{x}$ axis $\mathrm{X}_{\phi}=e^{-i 2 \pi \frac{\phi}{4 \sqrt{3}}\left[\left(\boldsymbol{\sigma}_{1} \cdot \boldsymbol{\sigma}_{2}-\mathbf{1}\right)-\left(\boldsymbol{\sigma}_{2} \cdot \boldsymbol{\sigma}_{3}-\mathbf{1}\right)\right]}$, with $\phi=\sqrt{3} \Delta J / h$. In typical qubit manipulation protocols, $J$ is constant and large, while $\Delta J$ is rapidly driven around zero 17$] 18$

\section{INTERFACES BETWEEN SPIN QUBITS}

\section{A. Single-spin qubit and singlet-triplet qubit}

Fig. 1(a) shows a trio of singly-occupied QDs that encodes a single-spin qubit and a STQ. $\mathrm{QD}_{1}$ defines the single-spin qubit, with the qubit levels $\left\{\left|0^{\mathrm{L}}\right\rangle,\left|1^{\mathrm{L}}\right\rangle\right\} . \mathrm{QD}_{2}$ and $\mathrm{QD}_{3}$ define the $\mathrm{STQ}$, where the qubit levels are called $\left\{\left|0^{\mathrm{R}}\right\rangle,\left|1^{\mathrm{R}}\right\rangle\right\}$. A general Hamiltonian in this setup is

$$
\begin{aligned}
\mathcal{H}^{\mathrm{A}}= & \frac{J_{12}}{4}\left(\boldsymbol{\sigma}_{\mathbf{1}} \cdot \boldsymbol{\sigma}_{\mathbf{2}}-\mathbf{1}\right)+\frac{E_{z}}{2}\left(\sigma_{z, 1}+\sigma_{z, 2}+\sigma_{z, 3}\right) \\
& +\frac{\widetilde{E}_{z, 2}}{2} \sigma_{z, 2}+\frac{\widetilde{E}_{z, 3}}{2} \sigma_{z, 3} .
\end{aligned}
$$

$\mathrm{QD}_{1}$ and $\mathrm{QD}_{2}$ are coupled by the exchange coupling $J_{12}$ that is described by the first term in Eq. (1). The second term describes the global magnetic field $E_{z}$, and the last two terms are the deviations of the local magnetic fields at $\mathrm{QD}_{2}$ and $\mathrm{QD}_{3}$ from $E_{z}$. To construct entangling operations, $\frac{E_{z}}{2}\left(\sigma_{z, 1}+\sigma_{z, 2}+\sigma_{z, 3}\right)$ and $\frac{\widetilde{E}_{z, 3}}{2} \sigma_{z, 3}$ can be neglected because these terms commute with the remaining parts of Eq. (1), and they generate only irrelevant phases. 
(a) single-spin qubit $\left\{\left|0^{\llcorner}\right\rangle,\left|1^{\llcorner}\right\rangle\right\}$

STQ

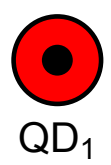
$\left\{\left|0^{R}\right\rangle,\left|1^{R}\right\rangle\right\}$

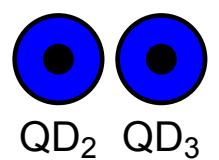

(b)

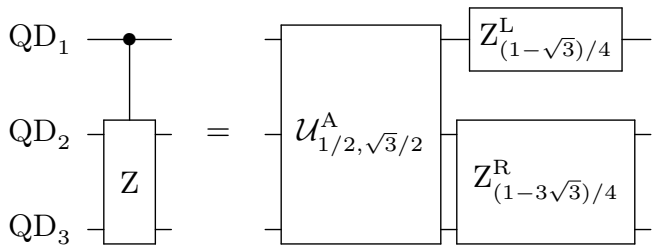

Figure 1. Entangling operation between a single-spin qubit and a STQ. (a) $\mathrm{QD}_{1}$ defines a single-spin qubit with the qubit levels $\left\{\left|0^{\mathrm{L}}\right\rangle,\left|1^{\mathrm{L}}\right\rangle\right\} ; \mathrm{QD}_{2}$ and $\mathrm{QD}_{3}$ define a STQ with the qubit levels $\left\{\left|0^{\mathrm{R}}\right\rangle,\left|1^{\mathrm{R}}\right\rangle\right\}$. A weak tunnel coupling between $\mathrm{QD}_{1}$ and $\mathrm{QD}_{2}$ couples the single-spin qubit and the STQ. (b) Sequence to create a CPHASE between a single-spin qubit (coded on $\mathrm{QD}_{1}$ ) and a STQ (coded on $\mathrm{QD}_{2}$ and $\left.\mathrm{QD}_{3}\right)$. $\mathrm{Z}_{\phi}^{\mathrm{L}}$ and $\mathrm{Z}_{\phi}^{\mathrm{R}}$ are the phase gates of the qubits $\mathrm{L}$ and R. $\mathcal{U}_{\phi, \psi}^{\mathrm{A}}$ is defined in Eq. (2).

The relevant time evolution is described by

$$
\mathcal{U}_{\phi, \psi}^{\mathrm{A}}=e^{-i 2 \pi\left[\frac{\phi}{4}\left(\boldsymbol{\sigma}_{\mathbf{1}} \cdot \boldsymbol{\sigma}_{\mathbf{2}}-\mathbf{1}\right)+\frac{\psi}{2} \sigma_{z, 2}\right]},
$$

with $\phi=J_{12} / h$ and $\psi=\widetilde{E}_{z, 2} / h$.

Only the states in the subspace $\left\{\left|0^{\mathrm{L}} 0^{\mathrm{R}}\right\rangle,\left|0^{\mathrm{L}} 1^{\mathrm{R}}\right\rangle,\left|1^{\mathrm{L}} 0^{\mathrm{R}}\right\rangle,\left|1^{\mathrm{L}} 1^{\mathrm{R}}\right\rangle,|\downarrow \uparrow \uparrow\rangle,|\uparrow \downarrow \downarrow\rangle\right\}$ are coupled in Eq. (2). There is no evolution from computational states to leakage states for $\sqrt{\phi^{2}+\psi^{2}}=\mathbb{Z}$. An entangling operation that is, up to local unitaries, equivalent to the CPHASE operation is realized for $\phi=\mathbb{Z}+\frac{1}{2}$. One can used, e.g., $\mathcal{U}_{1 / 2, \sqrt{3} / 2}^{\mathrm{A}}$. A CPHASE in the basis $\left\{\left|0^{\mathrm{L}} 0^{\mathrm{R}}\right\rangle,\left|0^{\mathrm{L}} 1^{\mathrm{R}}\right\rangle,\left|1^{\mathrm{L}} 0^{\mathrm{R}}\right\rangle,\left|1^{\mathrm{L}} 1^{\mathrm{R}}\right\rangle\right\}$ is [see Fig. 1 $\left.1(\mathrm{~b})\right]$ :

$$
\mathrm{Z}_{(1-\sqrt{3}) / 4}^{\mathrm{L}} \mathrm{Z}_{(1-3 \sqrt{3}) / 4}^{\mathrm{R}} \mathcal{U}_{1 / 2, \sqrt{3} / 2}^{\mathrm{A}}=e^{-i \frac{\pi}{2}} \text { CPHASE. }
$$

\section{B. Single-spin qubit and exchange-only qubit}

Fig. 2(a) shows a quartet of singly-occupied QDs that encodes a single-spin qubit $\left(\mathrm{QD}_{1} ;\right.$ qubit states $\left.\left\{\left|0^{\mathrm{L}}\right\rangle,\left|1^{\mathrm{L}}\right\rangle\right\}\right)$ and an exchange-only qubit $\left(\mathrm{QD}_{2}-\mathrm{QD}_{4}\right.$; qubit states $\left.\left\{\left|0^{\mathrm{R}}\right\rangle,\left|1^{\mathrm{R}}\right\rangle\right\}\right)$. A general interaction in this setup is

$$
\begin{aligned}
\mathcal{H}^{\mathrm{B}}= & \frac{J_{12}}{4}\left(\boldsymbol{\sigma}_{\mathbf{1}} \cdot \boldsymbol{\sigma}_{\mathbf{2}}-\mathbf{1}\right)+\frac{E_{z}}{2}\left(\sigma_{z, 1}+\sigma_{z, 2}+\sigma_{z, 3}+\sigma_{z, 4}\right) \\
& +\frac{J}{4}\left[\left(\boldsymbol{\sigma}_{\mathbf{2}} \cdot \boldsymbol{\sigma}_{\mathbf{3}}-\mathbf{1}\right)+\left(\boldsymbol{\sigma}_{\mathbf{3}} \cdot \boldsymbol{\sigma}_{\mathbf{4}}-\mathbf{1}\right)\right]
\end{aligned}
$$

The first term in Eq. (4) is the exchange coupling between $\mathrm{QD}_{1}$ and $\mathrm{QD}_{2}$. The second term is the global magnetic (a) single-spin qubit exchange-only qubit $\left\{\left|0^{\mathrm{L}}\right\rangle,\left|1^{\mathrm{L}}\right\rangle\right\} \quad\left\{\left|0^{\mathrm{R}}\right\rangle,\left|1^{\mathrm{R}}\right\rangle\right\}$

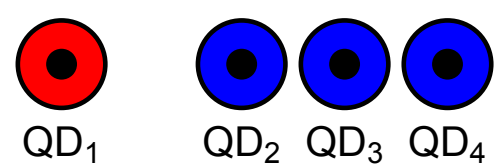

(b)

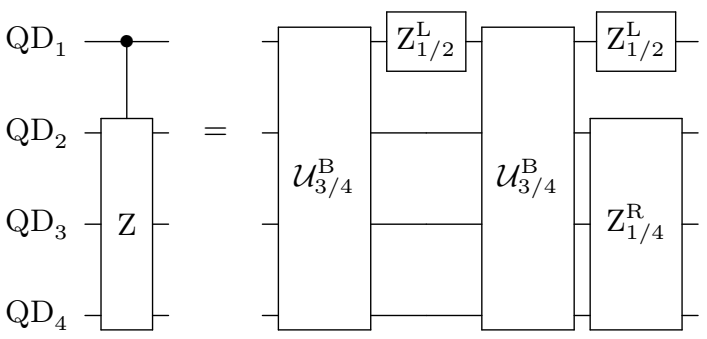

Figure 2. Entangling operation between a single-spin qubit and an exchange-only qubit. (a) $\mathrm{QD}_{1}$ defines a single-spin qubit with the qubit levels $\left\{\left|0^{\mathrm{L}}\right\rangle,\left|1^{\mathrm{L}}\right\rangle\right\} ; \mathrm{QD}_{2}-\mathrm{QD}_{4}$ define an exchange-only qubit with the qubit levels $\left\{\left|0^{\mathrm{R}}\right\rangle,\left|1^{\mathrm{R}}\right\rangle\right\}$. A weak tunnel coupling between $\mathrm{QD}_{1}$ and $\mathrm{QD}_{2}$ couples the single-spin qubit and the exchange-only qubit. (b) Sequence to create a CPHASE between a single-spin qubit (coded on $\left.\mathrm{QD}_{1}\right)$ and an exchange-only qubit (coded on $\left.\mathrm{QD}_{2}-\mathrm{QD}_{4}\right)$. $\mathrm{Z}_{\phi}^{\mathrm{L}}$ and $\mathrm{Z}_{\phi}^{\mathrm{R}}$ are the phase gates of the qubits $\mathrm{L}$ and R. $\mathcal{U}_{\phi}^{\mathrm{B}}$ is defined in Eq. (7).

field, and the third term describes the exchange couplings of the exchange-only qubit.

$\frac{E_{z}}{2}\left(\sigma_{z, 1}+\sigma_{z, 2}+\sigma_{z, 3}+\sigma_{z, 4}\right)$ commutes with the remaining parts of Eq. (4), and this term causes only an irrelevant time evolution of the single-spin qubit. The relevant time evolution through Eq. (4) is

$$
\mathcal{U}_{\phi, \psi}^{\mathrm{B}}=e^{-i 2 \pi\left\{\frac{\phi}{4}\left(\boldsymbol{\sigma}_{1} \cdot \boldsymbol{\sigma}_{2}-\mathbf{1}\right)+\frac{\psi}{4}\left[\left(\boldsymbol{\sigma}_{\mathbf{2}} \cdot \boldsymbol{\sigma}_{3}-\mathbf{1}\right)+\left(\boldsymbol{\sigma}_{\mathbf{3}} \cdot \boldsymbol{\sigma}_{4}-\mathbf{1}\right)\right]\right\}},
$$

with $\phi=J_{12} / h$ and $\psi=J / h$. There are exact entangling operations between a single-spin qubit and an exchangeonly qubit that use Eq. (5). However, these sequences are complicated and involve many operation steps $\frac{45}{4}$

Simpler entangling operations can be constructed for $J \gg J_{12}$. The computational subspace is part of the four-spin subspaces $S=0, s_{z}=0$ and $S=1$, $s_{z}=1,0$, which together have eight dimensions. ${ }^{46}$ Because the Hamiltonian in Eq. (4) preserves the spin quantum numbers, it is sufficient to describe the time evolution only in the four-spin subspaces $S=0$, $s_{z}=0$ and $S=1, s_{z}=1,0$ that are spanned by $\left\{\left|0^{\mathrm{L}} 0^{\mathrm{R}}\right\rangle,\left|0^{\mathrm{L}} 1^{\mathrm{R}}\right\rangle,\left|1^{\mathrm{L}} 0^{\mathrm{R}}\right\rangle,\left|l_{1}\right\rangle,\left|1^{\mathrm{L}} 1^{\mathrm{R}}\right\rangle,\left|l_{2}\right\rangle,\left|l_{3}\right\rangle,\left|l_{4}\right\rangle\right\}$, with $\left|l_{1}\right\rangle=\left|0^{\mathrm{L}}\right\rangle\left|u_{-1 / 2}\right\rangle, \quad\left|l_{2}\right\rangle=\left|0^{\mathrm{L}}\right\rangle\left|v_{-1 / 2}\right\rangle, \quad|l\rangle_{3} \propto$ $\left|0^{\mathrm{L}}\right\rangle\left|Q_{-1 / 2}\right\rangle-\left|1^{\mathrm{L}}\right\rangle\left|Q_{1 / 2}\right\rangle$, and $|l\rangle_{4} \propto \sqrt{3}\left|1^{\mathrm{L}}\right\rangle\left|Q_{3 / 2}\right\rangle-$ $\left|0^{\mathrm{L}}\right\rangle\left|Q_{1 / 2}\right\rangle$. The states $\left|u_{-1 / 2}\right\rangle=\frac{1}{\sqrt{2}}(|\uparrow \downarrow \downarrow\rangle-|\downarrow \downarrow \uparrow\rangle)$ and $\left|v_{-1 / 2}\right\rangle=\frac{1}{\sqrt{6}}(|\uparrow \downarrow \downarrow\rangle+|\downarrow \downarrow \uparrow\rangle)-\sqrt{\frac{2}{3}}|\downarrow \uparrow \downarrow\rangle$ span the $S=\frac{1}{2}, s_{z}=-\frac{1}{2}$ spin subspace of three electrons; $\left|Q_{3 / 2}\right\rangle=|\uparrow \uparrow \uparrow\rangle,\left|Q_{1 / 2}\right\rangle \propto|\uparrow \uparrow \downarrow\rangle+|\uparrow \downarrow \uparrow\rangle+|\downarrow \uparrow \uparrow\rangle,\left|Q_{-1 / 2}\right\rangle \propto$ $|\downarrow \downarrow \uparrow\rangle+|\downarrow \uparrow \downarrow\rangle+|\uparrow \downarrow \downarrow\rangle$, and $\left|Q_{-3 / 2}\right\rangle=|\downarrow \downarrow \downarrow\rangle$ are the $S=\frac{3}{2}$ 
quadruplet states of three spins. The spin labels corre- spond to $\left|\sigma_{\mathrm{QD}_{2}}, \sigma_{\mathrm{QD}_{3}}, \sigma_{\mathrm{QD}_{4}}\right\rangle$ in these state definitions.

The projection of Eq. (4) to the given basis is

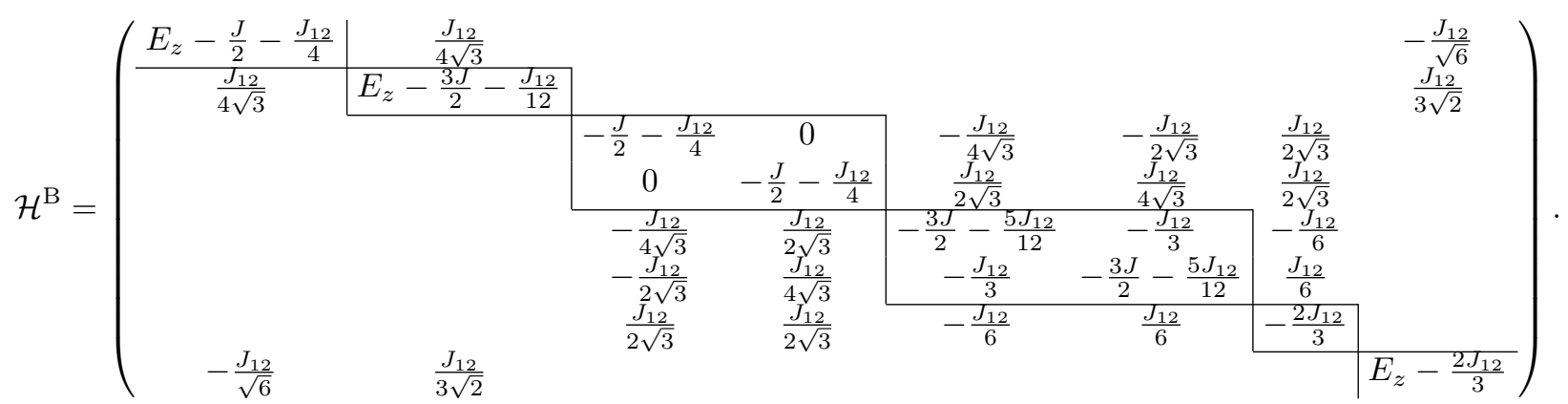

It is sufficient to consider the time evolution in the subspaces of equal energies that are defined by $E_{z}$ and $J$. The borders in the matrix of Eq. (6) indicate these subspaces. $J_{12}$ couples these subspaces, but for $E_{z}, J \gg J_{12}$ these processes can be neglected.

After neglecting all the entries outside of the marked subspaces in Eq. (6), also the time evolutions of $E_{z}$ and $J$ factor because they commute with the remaining entries. The global magnetic field $\frac{E_{z}}{2}\left(\sigma_{z, 1}+\sigma_{z, 2}+\right.$ $\left.\sigma_{z, 3}+\sigma_{z, 4}\right) \simeq \frac{E_{z}}{2}\left(\left|0^{\mathrm{L}}\right\rangle\left\langle 0^{\mathrm{L}}|-| 1^{\mathrm{L}}\right\rangle\left\langle 1^{\mathrm{L}}\right|\right)$ and the exchange interaction $\frac{J}{4}\left[\left(\boldsymbol{\sigma}_{2} \cdot \boldsymbol{\sigma}_{\mathbf{3}}-\mathbf{1}\right)+\left(\boldsymbol{\sigma}_{\mathbf{3}} \cdot \boldsymbol{\sigma}_{4}-\mathbf{1}\right)\right] \simeq$ $\frac{J}{2}\left(\left|0^{\mathrm{R}}\right\rangle\left\langle 0^{\mathrm{R}}|-| 1^{\mathrm{R}}\right\rangle\left\langle 1^{\mathrm{R}}\right|\right)$ cause single-qubit time evolutions that will be neglected in the following. Eq. (5) can then be simplified on the subspace $\left\{\left|0^{\mathrm{L}} 0^{\mathrm{R}}\right\rangle,\left|0^{\mathrm{L}} 1^{\mathrm{R}}\right\rangle,\left|1^{\mathrm{L}} 0^{\mathrm{R}}\right\rangle,\left|l_{1}\right\rangle,\left|1^{\mathrm{L}} 1^{\mathrm{R}}\right\rangle,\left|l_{2}\right\rangle\right\}$ to

$\mathcal{U}_{\phi}^{\mathrm{B}} \approx e^{-i 2 \pi \phi \operatorname{diag}}\left\{-\frac{1}{4},-\frac{1}{12},\left(\begin{array}{cc}-\frac{1}{4} & 0 \\ 0 & -\frac{1}{4}\end{array}\right),\left(\begin{array}{cc}-\frac{5}{12} & -\frac{1}{3} \\ -\frac{1}{3} & -\frac{5}{12}\end{array}\right)\right\}$.

$\operatorname{diag}\{a, b, \ldots\}$ describes the matrix with the diagonal entries $a, b, \ldots$, and $\phi=J_{12} / h$.

A single time evolution under Eq. (7) is never entangling because the criterion to prevent leakage only permits single-qubit gates. The two-step sequence $\mathcal{U}_{\phi}^{\mathrm{B}} Z_{1 / 2}^{\mathrm{L}} \mathcal{U}_{\phi}^{\mathrm{B}}$ is equivalent to a CPHASE gate for $\phi=\frac{3}{4}+\frac{3}{2} \mathbb{Z}$. A CPHASE operation in the basis $\left\{\left|0^{\mathrm{L}} 0^{\mathrm{R}}\right\rangle,\left|0^{\mathrm{L}} 1^{\mathrm{R}}\right\rangle,\left|1^{\mathrm{L}} 0^{\mathrm{R}}\right\rangle,\left|1^{\mathrm{L}} 1^{\mathrm{R}}\right\rangle\right\}$ is created by [see Fig. 2(b)]:

$$
\mathrm{Z}_{1 / 2}^{\mathrm{L}} \mathrm{Z}_{1 / 4}^{\mathrm{R}} \mathcal{U}_{3 / 4}^{\mathrm{B}} \mathrm{Z}_{1 / 2}^{\mathrm{L}} \mathcal{U}_{3 / 4}^{\mathrm{B}}=\text { CPHASE. }
$$

Note that the implicit single-qubit phase evolutions through $E_{z}$ and $J$, that are neglected in Eq. (7), need to be included in $\mathrm{Z}_{1 / 2}^{\mathrm{L}}$ and $\mathrm{Z}_{1 / 4}^{\mathrm{R}}$.

\section{Singlet-triplet qubit and exchange-only qubit}

A quintet of singly-occupied QDs, as shown in Fig. 3(a), defines a STQ $\left(\mathrm{QD}_{1}-\mathrm{QD}_{2} ;\right.$ qubit states $\left.\left\{\left|0^{\mathrm{L}}\right\rangle,\left|1^{\mathrm{L}}\right\rangle\right\}\right)$ and an exchange-only qubit $\left(\mathrm{QD}_{3}-\mathrm{QD}_{5}\right.$; qubit states $\left.\left\{\left|0^{\mathrm{R}}\right\rangle,\left|1^{\mathrm{R}}\right\rangle\right\}\right)$. A possible interaction in this setup is

$$
\begin{aligned}
\mathcal{H}^{\mathrm{C}_{1}=}= & \frac{J}{4}\left[\left(\boldsymbol{\sigma}_{\mathbf{3}} \cdot \boldsymbol{\sigma}_{\mathbf{4}}-\mathbf{1}\right)+\left(\boldsymbol{\sigma}_{\mathbf{4}} \cdot \boldsymbol{\sigma}_{\mathbf{5}}-\mathbf{1}\right)\right] \\
& +\frac{J_{23}}{4}\left(\boldsymbol{\sigma}_{2} \cdot \boldsymbol{\sigma}_{3}-\mathbf{1}\right)+\frac{\widetilde{E}_{z, 2}}{2} \sigma_{z, 2} \\
& +\frac{E_{z}}{2}\left(\sigma_{z, 1}+\sigma_{z, 2}+\sigma_{z, 3}+\sigma_{z, 4}+\sigma_{z, 5}\right) .
\end{aligned}
$$

The first term in Eq. (9) describes the single-qubit interaction of the exchange-only qubit for $J_{34}=J_{45}$, with the abbreviation $J=\left(J_{34}+J_{45}\right) / 2$. The second term is the exchange interaction between $\mathrm{QD}_{2}$ and $\mathrm{QD}_{3}$. A global magnetic field across all five QDs, $E_{z}$, is represented by the last term. $\widetilde{E}_{z, 2}$ is a small deviation of the local magnetic field at $\mathrm{QD}_{2}$ from the global magnetic field. Note that a possible deviation of the magnetic field at $\mathrm{QD}_{1}$, $\widetilde{E}_{z, 1}$, is irrelevant when the exchange interaction between $\mathrm{QD}_{1}$ and $\mathrm{QD}_{2}$ is reduced to zero. $\widetilde{E}_{z, 1}$ would only cause single-qubit evolutions of the STQ.

The time evolution under Eq. (9) can be used to construct an entangling operation between the STQ and the exchange-only qubit. Similar to the discussion in the previous section, $E_{z}$ and $J$ are much larger than $\widetilde{E}_{z, 2}$ and $J_{23}$. Therefore the qubit time evolution can be described using only the five-spin subspaces $S=\frac{1}{2}, s_{z}=\frac{1}{2}$ and $S=\frac{3}{2}, s_{z}=\frac{1}{2}$ that have together nine dimensions.

For $E_{z}, J \gg \widetilde{E}_{z, 2}, J_{23}$, only the states $\left|m_{1}\right\rangle=\left|T_{+}\right\rangle\left|u_{-1 / 2}\right\rangle$ and $\left|m_{2}\right\rangle=\left|T_{+}\right\rangle\left|v_{-1 / 2}\right\rangle$ coupled significantly to the computational subspace through Eq. (9). These states are eigenstates of $\frac{J}{4}\left[\left(\boldsymbol{\sigma}_{\mathbf{3}} \cdot \boldsymbol{\sigma}_{\mathbf{4}}-\mathbf{1}\right)+\left(\boldsymbol{\sigma}_{\mathbf{4}} \cdot \boldsymbol{\sigma}_{\mathbf{5}}-\mathbf{1}\right)\right]$, and they have identical energies as the qubit states. $\left|u_{-1 / 2}\right\rangle$ and $\left|v_{-1 / 2}\right\rangle$ 
span the $S=\frac{1}{2}, s_{z}=-\frac{1}{2}$ subspace of the spins at $\mathrm{QD}_{2}-\mathrm{QD}_{4}$ (using the definitions from Sec. IIIB). $m_{3}=$ $\sqrt{\frac{1}{2}}\left|T_{-}\right\rangle\left|Q_{3 / 2}\right\rangle-\sqrt{\frac{1}{3}}\left|T_{0}\right\rangle\left|Q_{1 / 2}\right\rangle+\sqrt{\frac{1}{6}}\left|T_{+}\right\rangle\left|Q_{-1 / 2}\right\rangle, m_{4}=$ $\sqrt{\frac{2}{5}}\left|T_{-}\right\rangle\left|Q_{3 / 2}\right\rangle+\sqrt{\frac{1}{15}}\left|T_{0}\right\rangle\left|Q_{1 / 2}\right\rangle-\sqrt{\frac{8}{15}}\left|T_{+}\right\rangle\left|Q_{-1 / 2}\right\rangle$, and $m_{5}=|S\rangle\left|Q_{3 / 2}\right\rangle$ have different energies, and therefore these states can be neglected. $\left|T_{+}\right\rangle=|\uparrow \uparrow\rangle,\left|T_{0}\right\rangle \propto|\uparrow \downarrow\rangle+|\downarrow \uparrow\rangle,\left|T_{-}\right\rangle=|\downarrow \downarrow\rangle$, and $\left|S_{0}\right\rangle=|\uparrow \downarrow\rangle-|\downarrow \uparrow\rangle$ are the usual triplet and singlet states at $\mathrm{QD}_{1}-\mathrm{QD}_{2}$. Projecting Eq. (9) to $\left\{\left|0^{\mathrm{L}} 0^{\mathrm{R}}\right\rangle,\left|1^{\mathrm{L}} 0^{\mathrm{R}}\right\rangle,\left|m_{1}\right\rangle,\left|0^{\mathrm{L}} 1^{\mathrm{R}}\right\rangle,\left|1^{\mathrm{L}} 1^{\mathrm{R}}\right\rangle,\left|m_{2}\right\rangle\right\}$ gives

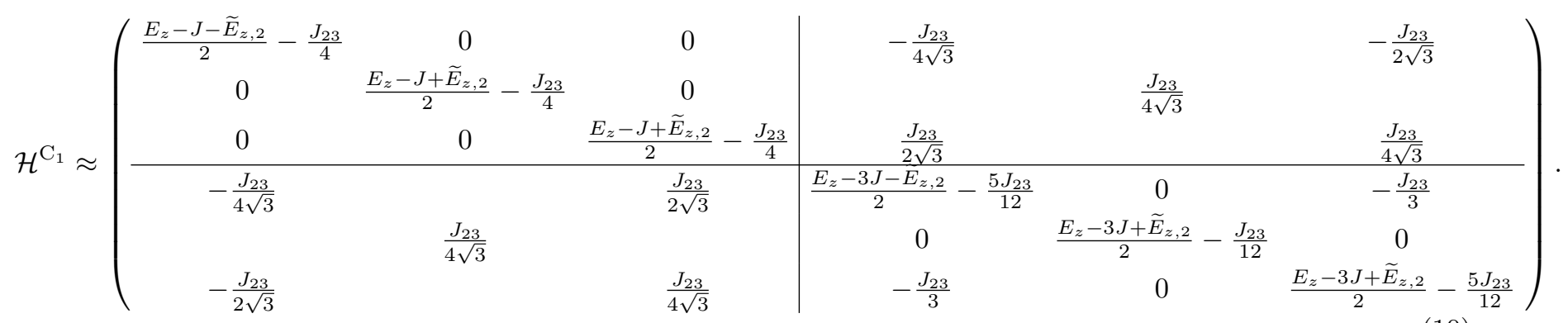

(10)
Eq. 10 contains two subspaces of virtually identical energies, as marked by the borders in the matrix. All the terms that couple these subspaces can be neglected.

After neglecting the block off diagonal entries in Eq. 10, , also the time evolutions of $E_{z}$ and $J$ factor because they commute with the remaining entries. The time evolution through $\frac{J}{4}\left[\left(\boldsymbol{\sigma}_{\mathbf{3}} \cdot \boldsymbol{\sigma}_{\mathbf{4}}-\mathbf{1}\right)+\left(\boldsymbol{\sigma}_{\mathbf{4}} \cdot \boldsymbol{\sigma}_{5}-\mathbf{1}\right)\right] \simeq$ $\frac{J}{2}\left(\left|0^{\mathrm{R}}\right\rangle\left\langle 0^{\mathrm{R}}|-| 1^{\mathrm{R}}\right\rangle\left\langle 1^{\mathrm{R}}\right|\right)$ causes only single-qubit time evolutions of the triple-QD qubit, and $E_{z}$ causes global phase evolutions. The remaining time evolution is

$$
\begin{aligned}
\mathcal{U}_{\phi, \psi}^{\mathrm{C}_{1}} & \approx e^{-i 2 \pi\left(\phi m_{1}+\frac{\psi}{2} m_{2}\right)}, \\
m_{1} & =-\operatorname{diag}\left\{\frac{1}{4}, \frac{1}{4}, \frac{1}{4},\left(\begin{array}{ccc}
\frac{5}{12} & 0 & \frac{1}{3} \\
0 & \frac{1}{12} & 0 \\
\frac{1}{3} & 0 & \frac{5}{12}
\end{array}\right)\right\}, \\
m_{2} & =\operatorname{diag}\{-1,1,1,-1,1,1\},
\end{aligned}
$$

with $\phi=J_{23} / h$ and $\psi=\widetilde{E}_{z, 2} / h$.

Eq. (11) causes no leakage for $\frac{1}{3} \sqrt{4 \phi^{2}+9 \psi^{2}}=$ $2 \mathbb{Z}+1$, and an entangling operation is realized for $\frac{1}{6}(2 \phi-3 \psi)=\mathbb{Z}$. Alternatively, it is also possible to use $\frac{1}{3} \sqrt{4 \phi^{2}+9 \psi^{2}}=2 \mathbb{Z}$ and $\frac{1}{6}(2 \phi-3 \psi)=\mathbb{Z}+\frac{1}{2}$. E.g., the entangling operation $\mathcal{U}_{3 /(2 \sqrt{2}), 1 / \sqrt{2}}^{\mathrm{C}_{1}}$ gives a CPHASE in the basis $\left\{\left|0^{\mathrm{L}} 0^{\mathrm{R}}\right\rangle,\left|0^{\mathrm{L}} 1^{\mathrm{R}}\right\rangle,\left|1^{\mathrm{L}} 0^{\mathrm{R}}\right\rangle,\left|1^{\mathrm{L}} 1^{\mathrm{R}}\right\rangle\right\}$ using [see
Fig. $3(\mathrm{~b})]$ :

$$
\mathrm{Z}_{1 / \sqrt{2}}^{\mathrm{L}} \mathrm{Z}_{(4+\sqrt{2}) / 8}^{\mathrm{R}} \mathcal{U}_{3 /(2 \sqrt{2}), 1 / \sqrt{2}}^{\mathrm{C}_{1}}=e^{i \pi \frac{\sqrt{2}-3}{2}} \text { CPHASE. }
$$

Note that in the construction of Eq. (12), it was assumed that $J_{12}$ is turned to zero during the entangling operation. Small values of $J_{12}$ can only be tolerated if they are much smaller than $\widetilde{E}_{z, 2}$. An alternative gate can be constructed for large $J_{12}$. In this case, Eq. $(9)$ is modified to

$$
\begin{aligned}
\mathcal{H}^{\mathrm{C}_{2}}= & \frac{J_{12}}{4}\left(\boldsymbol{\sigma}_{\mathbf{1}} \cdot \boldsymbol{\sigma}_{\mathbf{2}}-\mathbf{1}\right)+\frac{J}{4}\left[\left(\boldsymbol{\sigma}_{\mathbf{3}} \cdot \boldsymbol{\sigma}_{\mathbf{4}}-\mathbf{1}\right)+\left(\boldsymbol{\sigma}_{\mathbf{4}} \cdot \boldsymbol{\sigma}_{\mathbf{5}}-\mathbf{1}\right)\right] \\
& +\frac{\Sigma E_{z}}{2}\left(\sigma_{z, 1}+\sigma_{z, 2}\right)+\frac{J_{23}}{4}\left(\boldsymbol{\sigma}_{2} \cdot \boldsymbol{\sigma}_{3}-\mathbf{1}\right) \\
& +\frac{E_{z}}{2}\left(\sigma_{z, 1}+\sigma_{z, 2}+\sigma_{z, 3}+\sigma_{z, 4}+\sigma_{z, 5}\right)
\end{aligned}
$$

Eq. 13 contains the exchange interactions $J_{12}, J_{23}$, and $J$. Additionally to a global magnetic field $E_{z}$, the sum of the magnetic field variations at $\mathrm{QD}_{1}$ and $\mathrm{QD}_{2}$ are important $\Sigma E_{z}=\left(\widetilde{E}_{z, 1}+\widetilde{E}_{z, 2}\right) / 2$. The magnetic field difference $\Delta E_{z}=\left(\widetilde{E}_{z, 1}-\widetilde{E}_{z, 2}\right) / 2$ can be neglected if it is much smaller than $J_{12}$. Using the equivalent arguments as before for $E_{z}, J_{12}, J \gg \Sigma E_{z}, J_{23}$, the qubit time evolution is restricted to the subspace $\left\{\left|T_{0} 0^{\mathrm{R}}\right\rangle,\left|m_{1}\right\rangle,\left|T_{0} 1^{\mathrm{R}}\right\rangle,\left|m_{2}\right\rangle,\left|S_{0} 0^{\mathrm{R}}\right\rangle,\left|S_{0} 1^{\mathrm{R}}\right\rangle\right\}$. Projecting Eq. 13 to this basis gives 


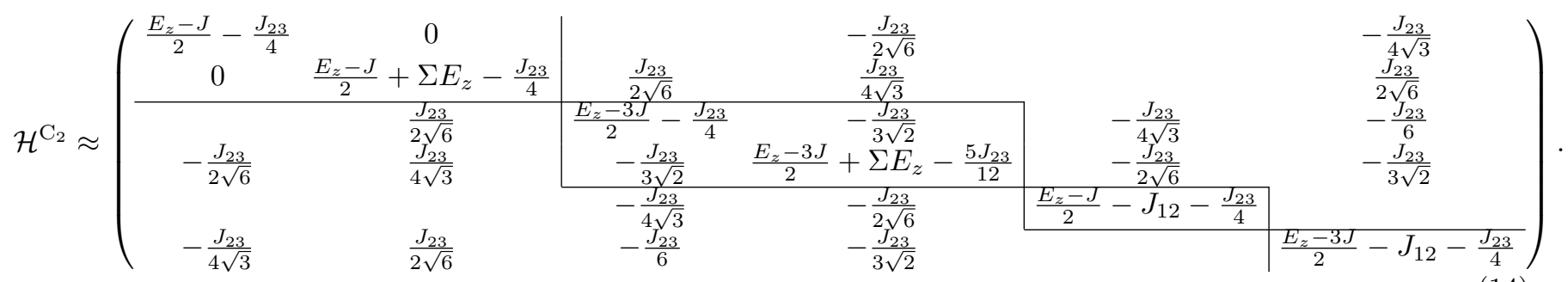

All the terms in Eq. 14 outside of the marked subspaces are neglected for $E_{z}, J_{12}, J \gg \Sigma E_{z}, J_{23}$. Neglecting the contributions of $E_{z}, J$, and $J_{12}$ (again these terms commute with the remaining entries in Eq. (14), and they cause either global phase evolutions, or single-qubit time evolutions) the effective time evolution is

$$
\begin{aligned}
\mathcal{U}_{\phi, \psi}^{\mathcal{C}_{2}} & \approx e^{-i 2 \pi\left(\phi m_{1}+\psi m_{2}\right)}, \\
m_{1} & =-\operatorname{diag}\left\{\left(\begin{array}{cc}
\frac{1}{4} & 0 \\
0 & \frac{1}{4}
\end{array}\right),\left(\begin{array}{cc}
\frac{1}{4} & \frac{1}{3 \sqrt{2}} \\
\frac{1}{3 \sqrt{2}} & \frac{5}{12}
\end{array}\right), \frac{1}{4}, \frac{1}{4}\right\}, \\
m_{2} & =\operatorname{diag}\{0,1,0,1,0,0\},
\end{aligned}
$$

with $\phi=J_{23} / h$ and $\psi=\Sigma E_{z} / h$. The contributions of $J_{12}$ and $J$ are irrelevant in Eq. (14) because only single-qubit time evolutions are generated: $\quad \frac{J_{12}}{4}\left(\boldsymbol{\sigma}_{1} \cdot \boldsymbol{\sigma}_{2}-\mathbf{1}\right) \simeq \frac{J_{12}}{2}\left(\left|0^{\mathrm{L}}\right\rangle\left\langle 0^{\mathrm{L}}|-| 1^{\mathrm{L}}\right\rangle\left\langle 1^{\mathrm{L}}\right|\right)$ and $\frac{J}{4}\left[\left(\boldsymbol{\sigma}_{\mathbf{3}} \cdot \boldsymbol{\sigma}_{\mathbf{4}}-\mathbf{1}\right)+\left(\boldsymbol{\sigma}_{\mathbf{4}} \cdot \boldsymbol{\sigma}_{\mathbf{5}}-\mathbf{1}\right)\right] \simeq \frac{J}{2}\left(\left|0^{\mathrm{R}}\right\rangle\left\langle 0^{\mathrm{R}}\right|-\right.$ $\left.\left|1^{\mathrm{R}}\right\rangle\left\langle 1^{\mathrm{R}}\right|\right)$. Also the phase evolution through $E_{z}$ is neglected.

The time evolution in Eq. (15) causes no leakage for $\frac{1}{2} \sqrt{\phi^{2}-\frac{4 \phi \psi}{3}+4 \psi^{2}}=2 \mathbb{Z}+1$, and an entangling operation is realized for $\frac{1}{12}(\phi-6 \psi)=\mathbb{Z}$. Alternatively, it is also possible to use $\frac{1}{2} \sqrt{\phi^{2}-\frac{4 \phi \psi}{3}+4 \psi^{2}}=2 \mathbb{Z}$ and $\frac{1}{12}(\phi-6 \psi)=\mathbb{Z}+\frac{1}{2}$. For example, the entangling operation $\mathcal{U}_{3 / \sqrt{2}, 1 /(2 \sqrt{2})}^{\mathrm{C}_{2}}$ gives a CPHASE gate in the basis $\left\{\left|0^{\mathrm{L}} 0^{\mathrm{R}}\right\rangle,\left|0^{\mathrm{L}} 1^{\mathrm{R}}\right\rangle,\left|1^{\mathrm{L}} 0^{\mathrm{R}}\right\rangle,\left|1^{\mathrm{L}} 1^{\mathrm{R}}\right\rangle\right\}$ using [see Fig. $3(\mathrm{c})$ ]:

$$
\mathrm{H}^{\mathrm{L}} \mathrm{Z}_{1 / 2}^{\mathrm{R}} \mathcal{U}_{3 / \sqrt{2}, 1 /(2 \sqrt{2})}^{\mathrm{C}_{2}} \mathrm{H}^{\mathrm{L}}=e^{i \pi \frac{3(\sqrt{2}-2)}{4}} \text { CPHASE, }
$$

where $\mathrm{H}$ is the Hadamard gate.

\section{DISCUSSION AND CONCLUSION}

It has been shown that the exchange interaction can be used to entangle a pair of QD qubits for all the distinct qubit encodings. Besides the single-qubit control, which has been experimentally realized for all the described spin qubits, only exchange interactions between a pair of QDs of different QD qubits are needed. With the flexibility of

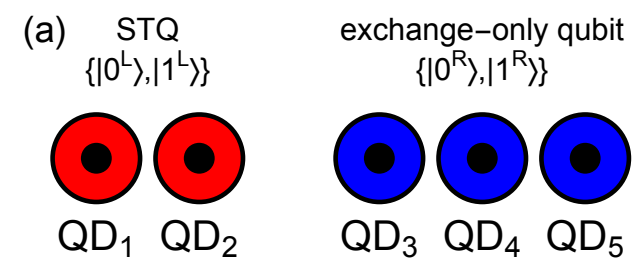

(b)

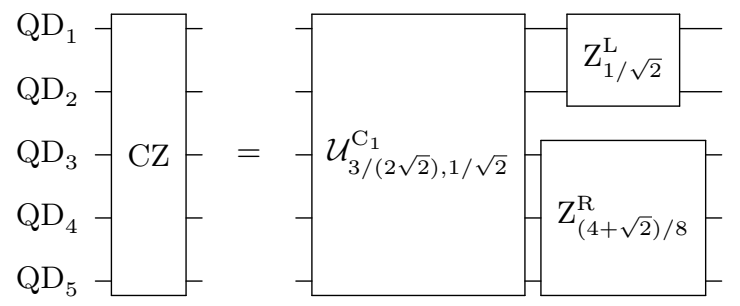

(c)

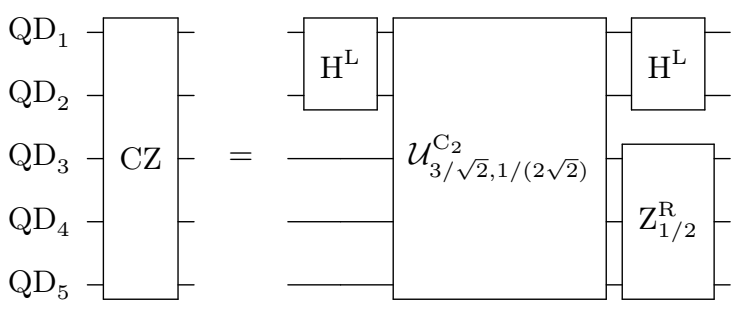

Figure 3. Entangling operations between a STQ and an exchange-only qubit. $\mathrm{QD}_{1}$ and $\mathrm{QD}_{2}$ define a $\mathrm{STQ}$ with the qubit levels $\left\{\left|0^{\mathrm{L}}\right\rangle,\left|1^{\mathrm{L}}\right\rangle\right\}$; $\mathrm{QD}_{3}-\mathrm{QD}_{5}$ define an exchange-only qubit with the qubit levels $\left\{\left|0^{\mathrm{R}}\right\rangle,\left|1^{\mathrm{R}}\right\rangle\right\}$. A weak tunnel coupling between $\mathrm{QD}_{2}$ and $\mathrm{QD}_{3}$ couples the $\mathrm{STQ}$ and the exchange-only qubit. (b)(c) Sequences to create a CPHASE between a STQ (coded on $\mathrm{QD}_{1}$ and $\mathrm{QD}_{2}$ ) and an exchangeonly qubit (coded on $\left.\mathrm{QD}_{3}-\mathrm{QD}_{5}\right)$. $\mathrm{Z}_{\phi}^{\mathrm{L}}$ and $\mathrm{Z}_{\phi}^{\mathrm{R}}$ are the phase gates of the qubits $\mathrm{L}$ and R. $\mathcal{U}_{\phi, \psi}^{\mathrm{C}_{1}}$ and $\mathcal{U}_{\phi, \psi}^{\mathrm{C}_{2}}$ are defined in Eq. (11) and Eq. 15. The CPHASE gate is abbreviated as $\mathrm{CZ}$, and $\mathrm{H}^{\mathrm{L}}$ is the Hadamard gate for qubit $\mathrm{L}$.

the spin qubit setup, i.e. by keeping constant exchange interactions (for the STQ or the exchange-only qubit) or allowing local magnetic field variations (for the STQ), very short operation sequences can be constructed to entangle QD qubits. To entangle a STQ with a single-spin qubit or an exchange-only qubit, only one exchange interaction is needed between QDs of the different qubit 


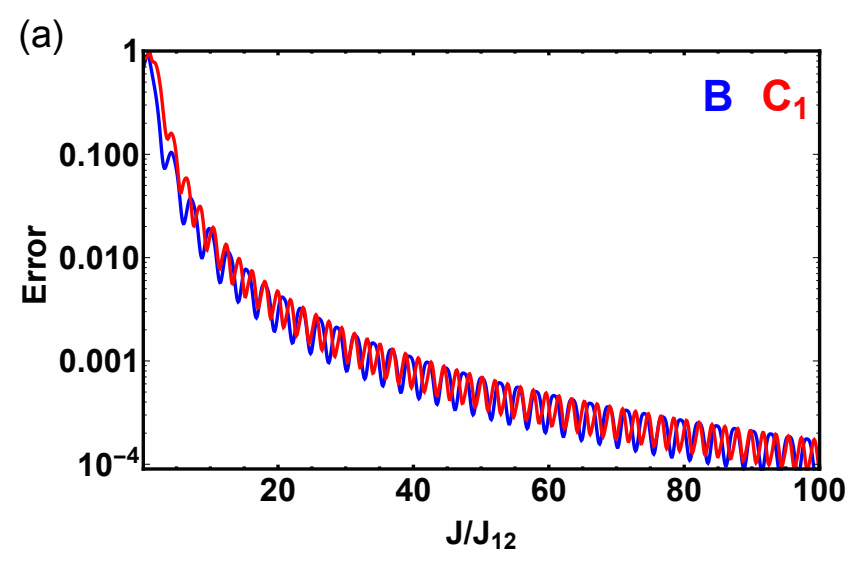

(b)

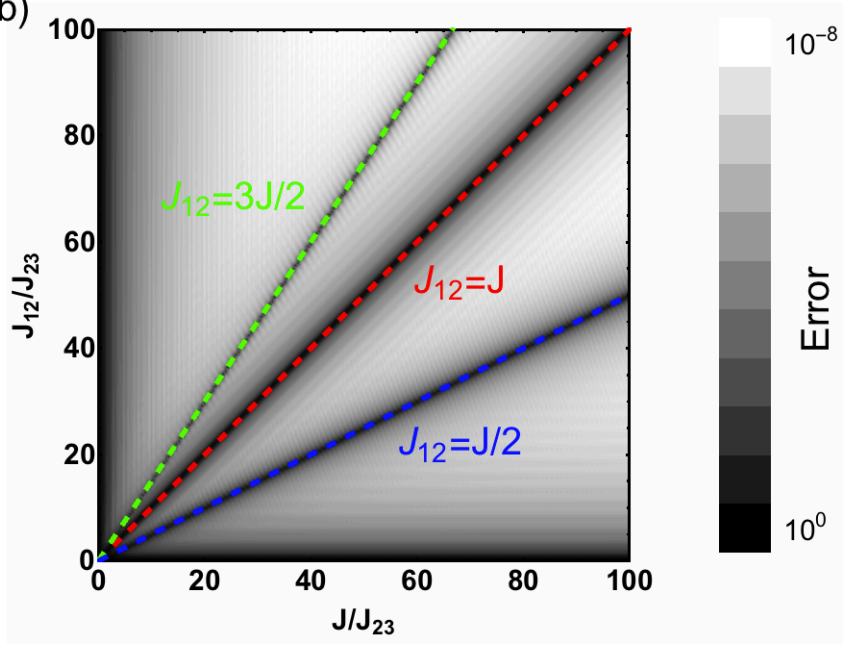

Figure 4. Gate errors for the operation sequences of Eq. 8), Eq. 12, and Eq. 16. Only the operations $\mathcal{U}_{3 / 4}^{\mathrm{B}}$, $\mathcal{U}_{3 /(2 \sqrt{2}), 1 / \sqrt{2}}^{\mathrm{C}_{1}}$, and $\mathcal{U}_{3 / \sqrt{2}, 1 /(2 \sqrt{2})}^{\mathrm{C}_{2}}$ are analyzed. The gate errors are characterized by the deviation of the entanglement fidelity $F=\operatorname{tr}\left(\rho^{\mathrm{RS}} \mathcal{U}_{\text {ideal }}^{-1} \mathcal{U}_{\text {real }} \rho^{\mathrm{RS}} \mathcal{U}_{\text {real }}^{-1} \mathcal{U}_{\text {ideal }}\right)$ from $1 . \rho^{\mathrm{RS}}=$ $|\mathrm{RS}\rangle\langle\mathrm{RS}|$ is the maximally entangled state of two identical subspaces R and S, e.g., $|\mathrm{RS}\rangle \propto|0000\rangle+|0110\rangle+|1001\rangle+$ $|1111\rangle$, and the time evolutions $\mathcal{U}_{\text {ideal }}$ and $\mathcal{U}_{\text {real }}$ act only on $\mathrm{S}$ while $\mathrm{R}$ remains unchanged. (a) For $\mathcal{U}_{3 / 4}^{\mathrm{B}}$ (blue curve) and $\mathcal{U}_{3 /(2 \sqrt{2}), 1 / \sqrt{2}}^{\mathrm{C}_{1}}$ (red curve) the exchange interaction of the exchange-only qubit $J$ should be by more than one order of magnitude larger than $J_{12}$ to reduce the gate error below $1 \%$. (b) For $\mathcal{U}_{3 / \sqrt{2}, 1 /(2 \sqrt{2})}^{\mathrm{C}_{2}}, J_{12}$ and $J$ should be large. The gate errors increase for $J_{12}=3 J / 2, J_{12}=J$, and $J_{12}=J / 2$ (dashed lines) because of degeneracies in the level spectrum.

types. To entangle a single-spin qubit and an exchangeonly qubit, a sequence of two inter-qubit exchange interactions is needed.

The advantage of exchange-based entangling operations is the controllability of the interaction mechanism. The exchange interaction depends on the tunnel coupling between distant QDs and their chemical potentials. It has been shown that exchange interactions can be tuned rapidly. ${ }^{43}$ The limitations of the proposed entangling operations are similar to existing gate schemes. (a)

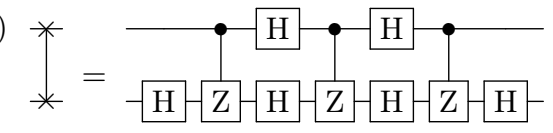

(b)

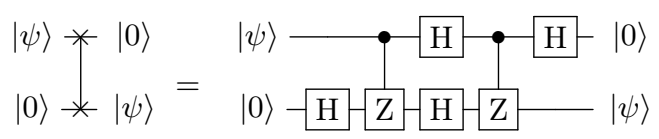

Figure 5. Gate operations to interchange qubits using CPHASE gates. (a) The unconditioned SWAP operation requires three CPHASE gates together with Hadamard gates (H). (b) A simpler SWAP sequence can be realized if one of the qubits is initialized to a fixed state, e.g., $|0\rangle$. Then the SWAP operation with an arbitrary state $|\psi\rangle$ requires only two CPHASE gates.

Local magnetic ${ }^{47}$ and electric field ${ }^{48}$ fluctuations are present in semiconductors. Both mechanism cause lowfrequency fluctuations of the QD parameters. It is possible to reduce the influence of low-frequency fluctuations by refocusing protocols, $\frac{49 \mid 50}{2}$ which can also be optimized numerically ${ }^{51}$ Spin-orbit interactions are weak in typical QD materials like, e.g., GaAs or Si,12 and they should have minor influence on the proposed operation sequences.

The constructions of the entangling operations in Sec. IIIB and Sec. IIIC used a few approximations. It was assumed that the exchange interaction between the QDs of the STQ, or between the QDs of the exchangeonly qubit are constantly turned on, while their magnitudes are much larger than the exchange interaction between the neighboring QDs of the different qubits. Fig. 4 show that about one order of magnitude difference in the interaction strength is sufficient to reduce the effective gate errors below 1\%. These gate errors are sufficient for quantum computation with standard quantum error correction protocols $[52,54$

Besides entangling different kinds of spin qubits, it might also be useful to interchange quantum information between them. Fig. 5 shows operation sequences for SWAP operations that only rely on CPHASE and Hadamard gates (cf. Ref. 55]). An unconditioned SWAP is realized using three CPHASE gates; only two CPHASE gates are needed if the state of a qubit should be transferred to another qubit that is initially in $|0\rangle$.

Altogether, very efficient operation sequences have been constructed to couple and interconvert different kinds of spin qubits. These operation sequences can couple all the standard qubit encodings in one, two, and three singly occupied QDs. Only the established singlequbit manipulation protocols are needed that have been successfully realized for all the qubit encodings. Different qubits are coupled using exchange interactions that are well controlled experimentally. With the current efforts to build larger arrays of tunnel-coupled QDs, the proposed operation sequences can be tested directly. The interconversion of different spin qubits allows to use 
all the advantages of the different QD setups in large arrays of QDs. E.g., it is known that few-electron qubits couple stronger to cavities $\frac{17|23| 24}{12}$ or metallic gates, $\frac{58}{,}$ while single-spin qubits have extremely long coherence times $\frac{8131}{21}$ Therefore the described operation sequences are another useful ingredient on the way towards quan- tum computation with large QD networks.

\section{ACKNOWLEDGMENTS}

We are grateful for support from the Alexander von Humboldt foundation.
* s.mehl@fz-juelich.de

1 R. Hanson, L. P. Kouwenhoven, J. R. Petta, S. Tarucha, and L. M. K. Vandersypen, "Spins in few-electron quantum dots," Rev. Mod. Phys. 79, 1217 (2007)

${ }^{2}$ F. A. Zwanenburg, A. S. Dzurak, A. Morello, M. Y. Simmons, L. C. L. Hollenberg, G. Klimeck, S. Rogge, S. N. Coppersmith, and M. A. Eriksson, "Silicon quantum electronics," Rev. Mod. Phys. 85, 961 (2013).

3 D. Loss and D. P. DiVincenzo, "Quantum computation with quantum dots," Phys. Rev. A 57, 120 (1998)

4 J. Levy, "Universal Quantum Computation with Spin-1/2 Pairs and Heisenberg Exchange," Phys. Rev. Lett. 89, $147902(2002)$

${ }^{5}$ D. P. DiVincenzo, D. Bacon, J. Kempe, G. Burkard, and K. B. Whaley, "Universal quantum computation with the exchange interaction," Nature (London) 408, 339 (2000).

6 E. Kawakami, P. Scarlino, D. R. Ward, F. R. Braakman, D. E. Savage, M. G. Lagally, M. Friesen, S. N. Coppersmith, M. A. Eriksson, and L. M. K. Vandersypen, "Electrical control of a long-lived spin qubit in a $\mathrm{Si} / \mathrm{SiGe}$ quantum dot," Nat. Nanotechnol. 9, 666 (2014).

7 P. Scarlino, E. Kawakami, D. R. Ward, D. E. Savage, M. G. Lagally, M. Friesen, S. N. Coppersmith, M. A. Eriksson, and L. M. K. Vandersypen, "Second Harmonic Coherent Driving of a Spin Qubit in a Si/SiGe Quantum Dot," arXiv:1504.06436 [cond-mat.mes-hall] (2015)

8 J. J. Pla, K. Y. Tan, J. P. Dehollain, W. H. Lim, J. J. L. Morton, D. N. Jamieson, A. S. Dzurak, and A. Morello, "A single-atom electron spin qubit in silicon," Nature (London) 489, 541 (2012)

${ }^{9}$ M. Veldhorst, J. C. C. Hwang, C. H. Yang, A. W. Leenstra, B. de Ronde, J. P. Dehollain, J. T. Muhonen, F. E. Hudson, K. M. Itoh, A. Morello, and A. S. Dzurak, "An addressable quantum dot qubit with fault-tolerant controlfidelity," Nat. Nanotechnol. 9, 981 (2014).

10 M. Veldhorst, R. Ruskov, C. H. Yang, J. C. C. Hwang, F. E. Hudson, M. E. Flatté, C. Tahan, K. M. Itoh, A. Morello, and A. S. Dzurak, "Spin-orbit coupling and operation of multi-valley spin qubits," arXiv:1504.06436 [cond-mat.mes-hall] (2015)

${ }^{11}$ R. Hanson and G. Burkard, "Universal Set of Quantum Gates for Double-Dot Spin Qubits with Fixed Interdot Coupling," Phys. Rev. Lett. 98, 050502 (2007).

12 S. Foletti, H. Bluhm, D. Mahalu, V. Umansky, and A. Yacoby, "Universal quantum control of two-electron spin quantum bits using dynamic nuclear polarization," Nat. Phys. 5, 903 (2009)

${ }^{13}$ H. Bluhm, S. Foletti, D. Mahalu, V. Umansky, and A. Yacoby, "Enhancing the Coherence of a Spin Qubit by Operating it as a Feedback Loop That Controls its Nuclear Spin Bath," Phys. Rev. Lett. 105, 216803 (2010).

14 X. Wu, D. R. Ward, J. R. Prance, D. Kim, J. K. Gam- ble, R. T. Mohr, Z. Shi, D. E. Savage, M. G. Lagally, M. Friesen, S. N. Coppersmith, and M. A. Eriksson, "Twoaxis control of a singlet-triplet qubit with an integrated micromagnet," Proc. Natl. Acad. Sci. 111, 11938 (2014).

15 J. Medford, J. Beil, J. M. Taylor, S. D. Bartlett, A. C. Doherty, E. I. Rashba, D. P. DiVincenzo, H. Lu, A. C. Gossard, and C. M. Marcus, "Self-consistent measurement and state tomography of an exchange-only spin qubit," Nat. Nanotechnol. 8, 654 (2013).

${ }^{10}$ K. Eng, T. D. Ladd, A. Smith, M. G. Borselli, A. A. Kiselev, B. H. Fong, K. S. Holabird, T. M. Hazard, B. Huang, P. W. Deelman, I. Milosavljevic, A. E. Schmitz, R. S. Ross, M. F. Gyure, and A. T. Hunter, "Isotopically enhanced triple-quantum-dot qubit," Sci. Adv. 1, e1500214 (2015).

17 J. M. Taylor, V. Srinivasa, and J. Medford, "Electrically Protected Resonant Exchange Qubits in Triple Quantum Dots," Phys. Rev. Lett. 111, 050502 (2013)

18 J. Medford, J. Beil, J. M. Taylor, E. I. Rashba, H. Lu, A. C. Gossard, and C. M. Marcus, "Quantum-Dot-Based Resonant Exchange Qubit," Phys. Rev. Lett. 111, 050501 (2013)

19 S. Mehl, H. Bluhm, and D. P. DiVincenzo, "Two-qubit couplings of singlet-triplet qubits mediated by one quantum state," Phys. Rev. B 90, 045404 (2014).

20 A. C. Doherty and M. P. Wardrop, "Two-Qubit Gates for Resonant Exchange Qubits," Phys. Rev. Lett. 111, 050503 (2013)

${ }^{21}$ M. Veldhorst, C. H. Yang, J. C. C. Hwang, W. Huang, J. P. Dehollain, J. T. Muhonen, S. Simmons, A. Laucht, F. E. Hudson, K. M. Itoh, A. Morello, and A. S. Dzurak, "A Two Qubit Logic Gate in Silicon," arXiv:1411.5760 [condmat.mes-hall] (2014)

${ }_{22}$ J. M. Taylor, H.-A. Engel, W. Dür, A. Yacoby, C. M. Marcus, P. Zoller, and M. D. Lukin, "Fault-tolerant architecture for quantum computation using electrically controlled semiconductor spins," Nat. Phys. 1, 177 (2005).

23 J. M. Taylor and M. D. Lukin, "Cavity quantum electrodynamics with semiconductor double-dot molecules on a chip," arXiv:cond-mat/0605144 [cond-mat.mes-hall] (2006)

${ }^{24}$ G. Burkard and A. Imamoglu, "Ultra-long-distance interaction between spin qubits," Phys. Rev. B 74, 041307 (2006)

25 M. D. Shulman, S. P. Harvey, J. M. Nichol, S. D. Bartlett, A. C. Doherty, V. Umansky, and A. Yacoby, "Suppressing qubit dephasing using real-time Hamiltonian estimation," Nat. Commun. 5, 5156 (2014)

26 T. Frey, P. J. Leek, M. Beck, A. Blais, T. Ihn, K. Ensslin, and A. Wallraff, "Dipole Coupling of a Double Quantum Dot to a Microwave Resonator," Phys. Rev. Lett. 108, 046807 (2012)

${ }^{27}$ K. D. Petersson, L. W. McFaul, M. D. Schroer, M. Jung, 
J. M. Taylor, A. A. Houck, and J. R. Petta, "Circuit quantum electrodynamics with a spin qubit," Nature (London) 490, 380 (2012).

${ }^{28}$ H. Toida, T. Nakajima, and S. Komiyama, "Vacuum Rabi Splitting in a Semiconductor Circuit QED System," Phys. Rev. Lett. 110, 066802 (2013)

${ }^{29}$ Y. S. Weinstein and C. S. Hellberg, "Energetic suppression of decoherence in exchange-only quantum computation," Phys. Rev. A 72, 022319 (2005).

30 S. Mehl, H. Bluhm, and D. P. DiVincenzo, "Fault-tolerant quantum computation for singlet-triplet qubits with leakage errors," Phys. Rev. B 91, 085419 (2015)

31 G. Balasubramanian, P. Neumann, D. Twitchen, M. Markham, R. Kolesov, N. Mizuochi, J. Isoya, J. Achard, J. Beck, J. Tissler, V. Jacques P. R. Hemmer, F. Jelezko, and J. Wrachtrup, "Ultralong spin coherence time in isotopically engineered diamond," Nature Mater. 8, 383 (2009).

32 B. E. Kane, "A silicon-based nuclear spin quantum computer," Nature (London) 393, 133 (1998).

33 J. T. Muhonen, J. P. Dehollain, A. Laucht, F. E. Hudson, T. Sekiguchi, K. M. Itoh, D. N. Jamieson, J. C. McCallum, A. S. Dzurak, and A. Morello, "Storing quantum information for 30 seconds in a nanoelectronic device," Nat. Nanotechnol. 9, 986 (2014)

${ }^{4} 4$ J. P. Dehollain, J. T. Muhonen, K. Y. Tan, A. Saraiva, D. N. Jamieson, A. S. Dzurak, and A. Morello, "SingleShot Readout and Relaxation of Singlet and Triplet States in Exchange-Coupled ${ }^{31} \mathrm{P}$ Electron Spins in Silicon," Phys. Rev. Lett. 112, 236801 (2014)

so J. T. Muhonen, A. Laucht, S. Simmons, J. P. Dehollain, R. Kalra, F. E. Hudson, S. Freer, K. M. Itoh, D. N. Jamieson, J. C. McCallum, A. S. Dzurak, and A. Morello, "Quantifying the quantum gate fidelity of single-atom spin qubits in silicon by randomized benchmarking," J. Phys.: Condens. Matter 27, 154205 (2015).

${ }^{30}$ M. Urdampilleta, A. Chatterjee, C. C. Lo, T. Kobayashi, J. Mansir, S. Barraud, A. C. Betz, S. Rogge, M. F. Gonzalez-Zalba, and J. J. L. Morton, "Charge dynamics and spin blockade in a hybrid double quantum dot in silicon," arXiv:1503.01049 [cond-mat.mes-hall] (2015).

37 R. H. Foote, D. R. Ward, J. R. Prance, J. K. Gamble, E. Nielsen, B. Thorgrimsson, D. E. Savage, A. L. Saraiva, M. Friesen, S. N. Coppersmith, and M. A. Eriksson, "Transport through an impurity tunnel coupled to a Si/SiGe quantum dot," arXiv:1505.02132 [cond-mat.meshall] (2015).

${ }^{38}$ F. H. L. Koppens, C. Buizert, K.-J. Tielrooij, I. T. Vink, K. C. Nowack, T. Meunier, L. P. Kouwenhoven, and L. M. K. Vandersypen, "Driven coherent oscillations of a single electron spin in a quantum dot," Nature (London) 442, 766 (2006)

${ }^{39}$ K. C. Nowack, F. H. L. Koppens, Y. V. Nazarov, and L. M. K. Vandersypen, "Coherent Control of a Single Electron Spin with Electric Fields," Science 318, 1430 (2007).

40 R. Brunner, Y.-S. Shin, T. Obata, M. Pioro-Ladrière, T. Kubo, K. Yoshida, T. Taniyama, Y. Tokura, and S. Tarucha, "Two-Qubit Gate of Combined Single-Spin Rotation and Interdot Spin Exchange in a Double Quantum Dot," Phys. Rev. Lett. 107, 146801 (2011)

41 J. Yoneda, T. Otsuka, T. Nakajima, T. Takakura, T. Obata, M. Pioro-Ladrière, H. Lu, C. Palmstrøm, A. C. Gossard, and S. Tarucha, "Fast Electrical Control of Single Electron Spins in Quantum Dots with Vanishing Influence from Nuclear Spins," Phys. Rev. Lett. 113, 267601 (2014).

${ }^{42}$ In contrast to Ref. [55], all the phase accumulations are given in multiples of $2 \pi$.

43 J. R. Petta, A. C. Johnson, J. M. Taylor, E. A. Laird, A. Yacoby, M. D. Lukin, C. M. Marcus, M. P. Hanson, and A. C. Gossard, "Coherent Manipulation of Coupled Electron Spins in Semiconductor Quantum Dots," Science 309, 2180 (2005)

44 S. Mehl and D. P. DiVincenzo, "Noise analysis of qubits implemented in triple quantum dot systems in a Davies master equation approach," Phys. Rev. B 87, 195309 (2013).

45 I found operation sequences to create entangling operations with a numerical search algorithm, similar to the description in Ref. [19. An operation sequence that is equivalent to a CPHASE is

$$
\mathcal{U}_{\phi_{1}, \phi_{2}}^{\mathrm{B}} \mathrm{X}_{\phi_{3}}^{\mathrm{R}} \mathcal{U}_{\phi_{1}, \phi_{2}}^{\mathrm{B}} \mathrm{Z}_{1 / 2}^{\mathrm{L}} \mathrm{X}_{\phi_{4}}^{\mathrm{R}} \mathcal{U}_{\phi_{1}, \phi_{2}}^{\mathrm{B}} \mathrm{X}_{\phi_{3}}^{\mathrm{R}} \mathcal{U}_{\phi_{1}, \phi_{2}}^{\mathrm{B}},
$$

with $\phi_{1}=0.195613200942698, \phi_{2}=0.2178346646839128$, $\phi_{3}=0.7362256575556158$, and $\phi_{4}=0.735072280195903$.

46 See the standard spin addition rules, e.g., in J. J. Sakurai and S. F. Tuan, Modern Quantum Mechanics (AddisonWesley, Reading, 1994).

47 G. Burkard, D. Loss, and D. P. DiVincenzo, "Coupled quantum dots as quantum gates," Phys. Rev. B 59, 2070 (1999)

$48 \mathrm{X} . \mathrm{Hu}$ and S. Das Sarma, "Charge-Fluctuation-Induced Dephasing of Exchange-Coupled Spin Qubits," Phys. Rev. Lett. 96, 100501 (2006)

${ }^{49}$ H Bluhm, S. Foletti, I. Neder, M. Rudner, D. Mahalu, V. Umansky, and A. Yacoby, "Dephasing time of GaAs electron-spin qubits coupled to a nuclear bath exceeding $200 \mu$ s," Nat. Phys. 7, 109 (2011).

50 J. Medford, L. Cywinski, C. Barthel, C. M. Marcus, M. P. Hanson, and A. C. Gossard, "Scaling of Dynamical Decoupling for Spin Qubits," Phys. Rev. Lett. 108, 086802 (2012)

5i P. Cerfontaine, T. Botzem, D. P. DiVincenzo, and H. Bluhm, "High-Fidelity Single-Qubit Gates for TwoElectron Spin Qubits in GaAs," Phys. Rev. Lett. 113, 150501 (2014)

52 A. G. Fowler, A. M. Stephens, and P. Groszkowski, "Highthreshold universal quantum computation on the surface code," Phys. Rev. A 80, 052312 (2009)

53 A. G. Fowler, M. Mariantoni, J. M. Martinis, and A. N. Cleland, "Surface codes: Towards practical large-scale quantum computation," Phys. Rev. A 86, 032324 (2012).

54 N. C. Jones, R. Van Meter, A. G. Fowler, P. L. McMahon, J. Kim, T. D. Ladd, and Y. Yamamoto, "Layered Architecture for Quantum Computing," Phys. Rev. X 2, 031007 (2012)

55 M. A. Nielsen and I. L. Chuang, Quantum Computation and Quantum Information (Cambridge University Press, Cambridge, 2000).

56 T. Takakura, A. Noiri, T. Obata, T. Otsuka, J. Yoneda, K. Yoshida, and S. Tarucha, "Single to quadruple quantum dots with tunable tunnel couplings," Appl. Phys. Lett. 104, 113109 (2014).

${ }^{5}$ M. R. Delbecq, T. Nakajima, T. Otsuka, S. Amaha, J. D. Watson, M. J. Manfra, and S. Tarucha, "Full control of quadruple quantum dot circuit charge states in the single electron regime," Appl. Phys. Lett. 104, 183111 (2014)

58 L. Trifunovic, O. Dial, M. Trif, J. R. Wootton, R. Abebe, A. Yacoby, and D. Loss, "Long-Distance Spin-Spin Coupling via Floating Gates," Phys. Rev. X 2, 011006 (2012). 